\title{
Assessment of Service Departments in the Governorates Through Social Media Comments
}

\author{
Sura Sabah Rasheed ${ }^{1}$, Ahmed T. Sadiq ${ }^{2}$ \\ ${ }^{1,2}$ Department of Computer Science, University of Technology, Baghdad, Iraq. \\ ${ }^{1}$ suramaster2020@gmail.com, ${ }^{2}$ Ahmed.T.Sadiq@uotechnology.edu.iq
}

\begin{abstract}
Social media have been increasing obviously and widely due to the fact that it is a media for users who express their emotions using reviews and comments on a variety of areas in life. In the present study, a modest model has been suggested for the assessment of service departments with the use of reviews and comments in social media pages of those departments from various governorates. The utilization of the text mining for the sentiment classification has been used through collecting Iraqi dialect reviews on service department pages on Facebook to be analyzed with the use of the sentiment analysis to track the emotions from the comments and posts. Those have been classified after that to positive, neutral or negative comment with the use of the algorithms of Naive Bayesian, Rough Set Theory, and K-Nearest Neighbors. Out of 13 Iraqi capital (Baghdad) service departments have been tackled, it has been found that $11 \%$ of those departments had very good assessment, $18 \%$ from these service departments have good assessment, $21 \%$ from these service departments have medium assessment, 24\% from these service departments have acceptance assessment and $26 \%$ from these service departments have bad assessment. The results of the evaluation showed the poor services provided by service departments in the capital Baghdad. Experimental results were helpful for the service departments in improving their work and programs had responded quickly and sufficiently to the customer demands.
\end{abstract}

Index Terms—Facebook Data, Rough Set Theory, Naïve Bayesian, Sentiment Analysis, K-NN, Text Mining.

\section{Introduction}

Online websites suffer from the lags in the marketing of their products because of the lack in the effective systems analyzing and tracing the assessments of customers to their services; that is why some governmental are still unknown in spite of their good quality of service [1]. Social media like Facebook, Twitter and Instagram took a considerable part of the publics and actions as a result of the rapid and massive advances in the field of information technology. Numerous users use the communication sites for discovering new associates and sharing materials, as well as for showing their moods by different ways, like the electronic posting wall of comments. Therefore, the Arab 
countries have counted a massive amount of the social media users and have become a suitable media for endorsing freedom of speech. Therefore, this media made it a rich resource for the sentiment analysis and text mining approaches [2]. A very significant service in the social media is governmental service departments, which can be considered as a very dominant topic on Twitter and Facebook. The projects of the abovementioned departments affect the lives of millions of people in different governorates and in numerous aspects, such as electricity, communal services, municipal services, and many others [3]. Text Mining has been utilized for the detection of the undiscovered information on social media for obtaining useful information from a variety of the reviews and comments. In addition to that, web text mining utilizes the approaches of data mining for the automatic extraction and analysis of information for achieving knowledge discovery. Web data is usually unlabeled, heterogeneous, distributed, time varying, semi-structured, and high dimensional $[4,5]$. Two types of information flow in our application, the first flow is from the number of followers to service department Facebook pages and the other flow is the aggregate information from the citizens to the service department. These two flows of information may be provided and analyzed with the use of the proper approaches of text mining for exploring the vital and meaningful decisions for the numerous service departments [3]. The most significant part of the way of information collection is getting the thoughts and emotions of the other users. With the increasing popularity of the sites of on-line reviews, new problems have emerged for the use of the approaches of information extraction for the detection and understanding of others' sentiments [6].

\section{The main contributions of this study can be summarized as:}

1-The first contribution is the way of extracting comments available on Facebook pages and the way it may be utilized for drawing meaningful decisions on the governmental service departments.

2- The majority of algorithms are utilized for the classification of English text. A very limited number of the studies deal with the Arabic and Iraqi vernacular text classification due to the fact that it has affluent morphologies.

In the present study, a model of sentiment analysis for the Service Departments has been proposed, utilizing the Facebook posts written in Iraqi dialect. In section 2, relevant studies on this area have been subjected. In section 3, the general ideas of sentiment analysis have been discussed and processes are summed up. In section 4, the model that has been proposed will be presented. In section 5, the test on the proposed model is carried out. The final section includes conclusions that have been drawn from this study. 


\section{Related work}

Below are some the previous studies that are relevant to the approaches that have been utilized for sentiment analysis are presented:

1- In [2] (2013), this study had explored the methods of text mining for the sentiment analysis. Tunisian user statuses and behaviors have been extracted from Facebook posts throughout the period of "Arabic Spring” for the purpose of investigating the Tunisians' thoughts during the revolution in December and January at 2011. Classification methods that have been based on SVMs and Naïve Bayesian have been utilized. A sentiment lexicon, which has been based upon emoticons, acronyms and interjections have been constructed from the extracted status updates on Facebook. Comparative tests have been carried out between 2 approaches of machine learning methods, which are SVM and Naïve Bayes via a training model for classification of sentiments. It has been concluded that, using Naïve Bayes (NB), the accuracy had reached high in the case where bigram feature has been used. On the other hand, the SVM exhibited a better performance compared to Naïve Bayesian in the case of using the unigram features.

2- In [4] (2020), a conceptual model that has been based upon the text mining has been utilized for the classification of texts from emotional data-set to 4 emotional groups: anger, fear, joy, and sadness. The Naïve Bayes optimized by particle swarm optimization (PSO) has been used as classifier. The pre-processing steps within model development have been: document collection, transformation into lower cases, tokenization, token filtering (by length), removal of stop words, stemming, and vector creation. The experimental results have resulted in an accuracy about $65.93 \%$ with the use of the Naïve Bayesian without the optimization, and $66.54 \%$ with PSO which showed an increase in the accuracy.

3- In [6] (2013) sentiment analysis has been carried out on Arabic Facebook news pages. The suggested system included pre-processing, selections of feature vectors and classification methods using SVMs, Naive Bayesian and decision tree. 2400 comments have been collected and represented in a distinct record, and then grouped in 3 groups (supportive, neutral and attacking). After grouping, 800 represented neutral comments, 800 as supportive comments, and 800 as attacking comments. The accuracy reached about $59.90 \%$ using Naive Bayesian. The precision and recall are improved 10\% with the decision tree and finally SVM gave the best for precision and recall which is about $73.4 \%$.

4- In [7] (2018), an extraction has been carried out on a group of the reviews from the public page 'Opposing Views' on Facebook using the software QSR NVivo 11 that is utilized for the analysis of the unstructured data into positive or negative comments. The auto code feature in QSR NVivo 11 was utilized to analyze and tag the comments to positive or negative sentiments. 
Different Techniques were used for preprocessing such as Tokenization, Stemmed words and Query Augmentation for synonyms. After analyzing the specified number of comments, positive sentiments were about $29.6 \%$ and negative sentiments about $62.0 \%$. The experimental results shown that the percentage of negative reviews is twice more than positive reviews.

5- In [8] (2008), the authors have incorporated approaches of sentiment analysis for the analysis of the reviews from the travel blogs. Naive Bayesian, SVMs and N-gram character based are approaches of the sentiment classifications utilized for the classification of reviews from the travel blogs for seven common destinations in Europe and U.S. Experimental results have shown that when a large number of reviews have been analyzed, all three methodologies nearly approached a minimum of $80 \%$ accuracy.

6- In [9] (2017), an Arabic corpora has been built based on Facebook (FB) posts that have been written in Dialectal Arabic (DA) without grammar. The collections are labeled with 5 labels (positive, negative, dual, neutral, and spam). The words that specify the opinions have been utilized in lexicon-based classifier. It has been obtained an accuracy between $73 \%$ and $96 \%$, depending on the classical, modern and dialectal Arabic language.

7- In [10] (2016), a comparative study applied SVMs, NB and Multilayer Perceptron (MLP-NN) classification methods on Arabic datasets which are Aljazeera news website Saudi Press, Agency (SPA) and Alhayat. Experimental results have been applied on 1400 Arabic documents that belong to different categories had yielded a precision of approximately $0.778,0.754$, and 0.717 for SVMs, NB and MPL-NN respectively, with the use of 600 input layers.

\section{Sentiment Analysis}

Sentiment analysis (SA) has been presented first by Liu, B [11]. It has also been referred to as the opinion mining that can be defined as the procedure of the determination of polarity of the reviews or opinions that have been written by the humans for rating the services or products. SA may be performed on a document level, in which the whole text is evaluated for the determination of the polarity of opinions through feature extraction. In addition to that, it may be carried out on sentence level where text has been partitioned to sentences to be evaluated in a separate manner for the determination of the polarity of the documents. Text polarity may be negative, neutral or positive. In service departments, information has been given through comments that are either subjective (opinionated) i.e. based on personal feelings or decision making on the events or objective (factual) that are based upon information, opinions and evidences. The posts and comments of the citizens usually express pleasure, prevention, displeasure, enjoyment. This sentiment information has a considerable influential for the service departments that seek at enhancing the people's satisfaction 
and efficiency of work [12]. SA includes multistep operations, which are: a) data retrieval, b) extraction and selection of required data, c) preprocessing, d) extraction of features, e) subject detection, and f) application of data mining techniques [3]. There are 2 main approaches for the extraction of sentiments. The first one is by the use of the lexical-based analyses, involving the manipulation of document polarity from polarity of terms in documents. The second one is by the use of the approaches of Machine learning that are categorized to supervised and unsupervised approaches. The supervised method that has been implemented in the suggested model includes the classifier construction from the labelled text examples via a supervised classification procedure. The benefit of machine learning approaches is to create the trained models for the trained contents for the classification of new data. The key of machine learning approaches that are utilized for SA are SVMs and Naive Bayesian as they have been often designed for the tasks of binary classification [3, 13]. Rough set theory plays a big role in text classification and categorization within different areas [14, $15,16,17]$ therefore it is utilized in this paper as a classifier. Furthermore, Naïve Bayes Classifier (NBC) $[18,19]$ is also utilized in this paper as a probabilistic classifier, which uses Bayes' theorem as a decision rule with independent features.

\section{The Proposed Method}

The stages of the suggested model have been presented. The fundamental aim of the present study is assessing the service departments in different governorates based on the extraction off the sentiments from comments of users on the social networks of those departments for discovering useful decisions. Which is why, sentiment analysis of texts that are included in the comments is the basic operation in the proposed model. The following are the main steps of the proposed model and the diagram in Fig. (1) Illustrate the main steps.

1- Text Comments Collection Stage: In the first stage, the users are the source of information in social media, as they leave feedbacks about their opinion on the performance of different service departments. So, these text comments are collected for each department in a separate manner to be additionally processed.

2- The Preprocessing Stage: in this stage, a transformation has been implemented for transforming the extracted raw text comments into a suitable representation for the following stage. Which involves 3 processes, namely: tokenization process, stemming process and removal of the stop-words process. Lite stemming has been used in the proposed model. 


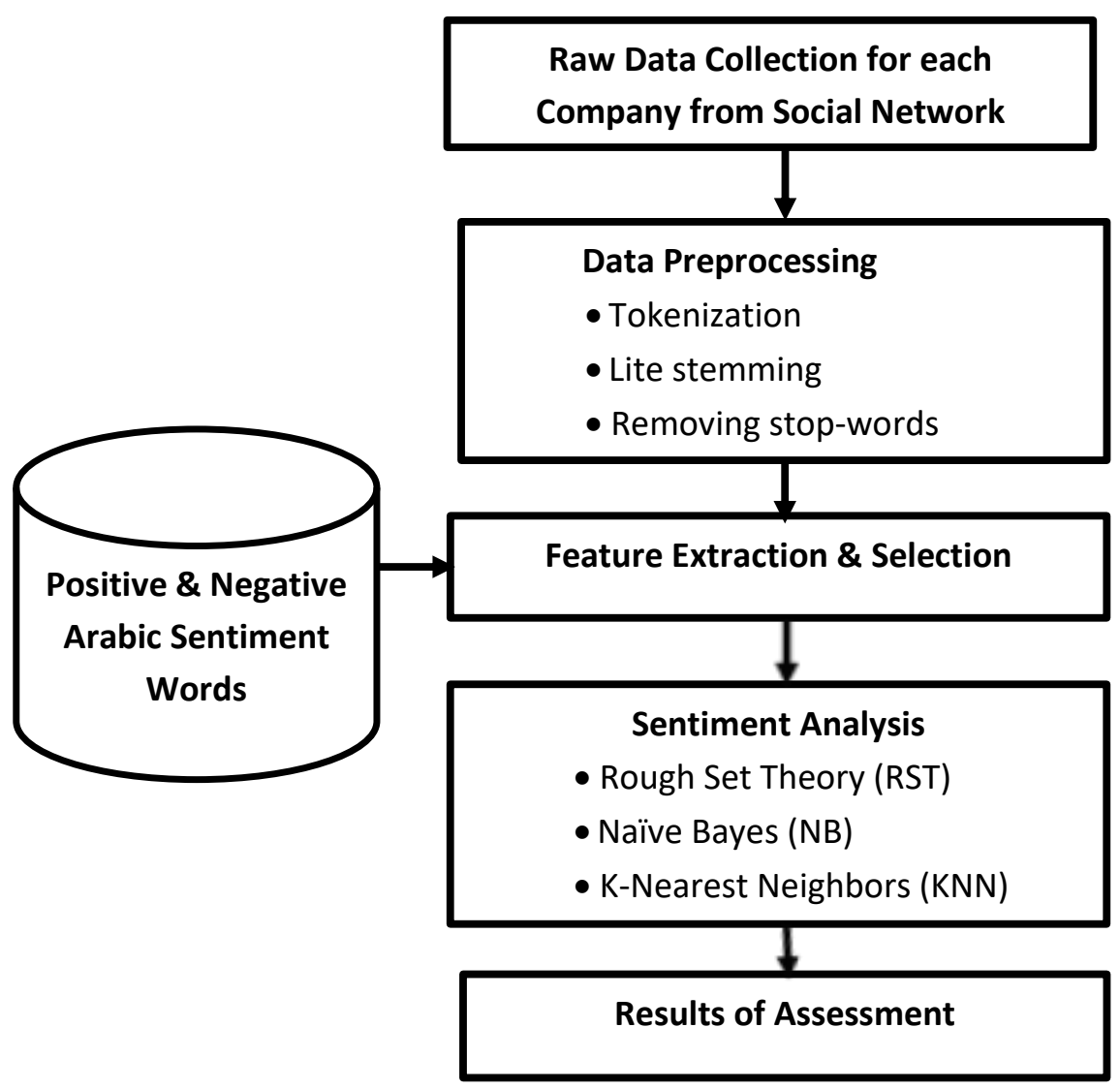

Fig. 1. The Main Steps of the Proposed Model

3- $\quad$ Features Extraction and Selection Stage: in this stage, sentiment words are chosen (i.e. verbs and adjectives) and annotated as positive and negative sentiments. As Iraqi Dialect includes all forms of spoken Arabic in daily life, there are classical Arabic words within Iraqi dialect which are positive and negative words [20]. Those words have an important impact on sentiment analysis and as a result on the assessment results of the effectiveness and quality. Two types of Arabic positive and negative words have been considered in this step, which are: single-word and double-word. It consists of 532 single-words (215 positive and 317 negative) and 419 double-words (233 positive and 186 negative).

4- Sentiment Analysis Stage: in this stage, 3 different machine learning algorithm types have been implemented in the steps of sentiment analysis, those are Rough Set Theory (RST), NB and KKNNs. Finally, based upon the results of sentiment analysis that are either positive or negative, the assessment is decided. 


\section{Example :}

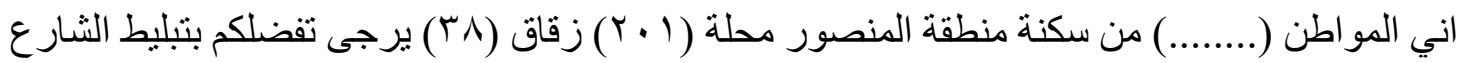

$$
\begin{aligned}
& \text { الفر عي لزقاقنا بسبب كثرة المطبات و الحفر مما يسبب تجمع المياه والاطيان في الثنار ع في فصل الثتاء. }
\end{aligned}
$$

\section{Result :}

Argument = " كثرة المطبات و الحفر مما يسبب تجمع المياه والاطيان في الثارع في فصل الثتاء"

\section{Experimental Results}

In this section, the proposed model is experimented with the information that has been collected from Facebook. The dataset have been collected from 13 pages of Iraqi capital (Baghdad) service departments in Facebook (officials and others), Table (1) shows the number of dataset details.

\section{TABle 1. Dataset Details}

\begin{tabular}{cccc} 
Number of Pages & Number of Posts & Number of comments & Number of Sentences \\
\hline 13 & 260 & 7800 & 33875
\end{tabular}

As mentioned in the table above, there are 13 department pages (officials and others) in Facebook, 20 posts from every one of those departments were taken, 30 comments from every post have been chosen, and the total sentences from all of the comments have been 15320 . The sentiment analysis took under consideration the classical Arabic language and Iraqi dialect words.

Three classification methods have been implemented for 7800 comments; those methods are Rough Set Theory, NB and KNNs. Figure (2) illustrates the classification methods' accuracy ratio for the sentiment analysis for the dataset above, where the accuracy ratio was calculated using Eq. (1) multiplication by 100 .

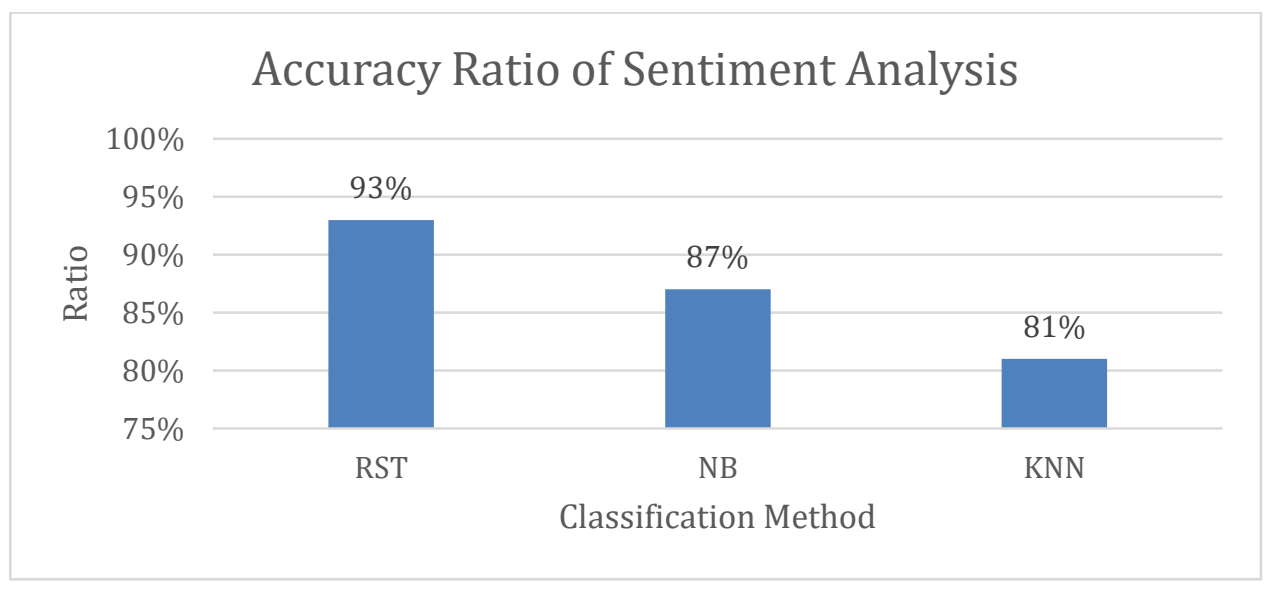

Figure 2. Sentiment Analysis Accuracy

Received 26/4/2021; Accepted 25/6/2021 
The best one is the Rough Set Theory (RST), Table (1) illustrates the confusion matrix of RST classification method.

Table (2) Multiclass Confusion Matrix for 7800 Comments' Sentiment Classification

\begin{tabular}{ccccc} 
& $\begin{array}{c}\text { Positive } \\
\text { Predicted } \\
\text { Class }\end{array}$ & $\begin{array}{c}\text { Negative } \\
\text { Predicted } \\
\text { Class }\end{array}$ & $\begin{array}{c}\text { Neutral } \\
\text { Predicted } \\
\text { Class }\end{array}$ & Total \\
\hline $\begin{array}{c}\text { Positive } \\
\text { Actual Class }\end{array}$ & 2651 & 149 & 50 & 2850 \\
$\begin{array}{c}\text { Negative } \\
\text { Actual Class } \\
\text { Neutral }\end{array}$ & 195 & 3734 & 86 & 4015 \\
Actual Class & 17 & 48 & 870 & 935 \\
& & & & 7800
\end{tabular}

In more details, below tables (3, 4 and 5) show the confusion matrices for the positive, negative and neutral sentiment of the comments respectively.

TABle (3) Confusion Matrix FOR Positive SEntiment

\begin{tabular}{lccc} 
& Positive & Non-Positive & Total \\
\hline Positive & 2651 & 199 & 2850 \\
Non-Positive & 346 & 4604 & 4950 \\
& & & 7800
\end{tabular}

TABle (4) CONFusion MAtriX FOR Negative SENTIMENT

\begin{tabular}{lccc} 
& Negative & Non-Negative & Total \\
\hline Negative & 3734 & 281 & 4015 \\
Non-Negative & 264 & 3521 & 3785 \\
& & & 7800
\end{tabular}

TABle (5) CONFusion MATRIX FOR NeUtRal SENTIMENT

\begin{tabular}{lccc} 
& Neutral & Non-Neutral & Total \\
\hline Neutral & 870 & 65 & 935 \\
Non-Neutral & 480 & 6385 & 6865 \\
& & & 7800
\end{tabular}

The accuracy equation for binary classifier is calculated as follows:

$$
\text { Accuracy }=\frac{T P+T N}{T P+F N+F P+T N} \ldots \ldots \ldots \text { (1) }
$$

Where TP is True Positive, TN is True Negative, FP is False Positive and FN is False Negative. 
From above Eq. (1) and using data from Table 3, 4 and 5 it could be shown that the accuracy reached about $93.01 \%$ on positive comments, whereas it reached about $93.2 \%$ and $92.8 \%$ on negative and neutral comments. Furthermore, the average accuracy which is the average of per-class effectiveness value of the classifier is calculated using the following Eq. (2). The resulted average accuracy is about $93.05 \%$.

$$
\text { Average Accuracy }=\frac{\sum_{i=1}^{l} \frac{T P_{i}+T N_{i}}{T P_{i}+F N_{i}+F P_{i}+T N_{i}}}{l} \ldots \ldots
$$

Where $l$ is total number of classes.

The average Error Rate which is the average of per-class classification error is computed using the following Eq. (3), which resulted about $95.6 \%$.

$$
\text { Average Error Rate }=\frac{\sum_{i=1}^{l} \frac{F P_{i}+F N_{i}}{T P_{i}+F N_{i}+F P_{i}+T N_{i}}}{l} \ldots \ldots \ldots
$$

The following equation Eq. (4) shows the Precision computation which computed the average perclass agreement of the human judgment with the classification model which yielded about $93.02 \%$.

$$
\text { Precision }=\frac{\sum_{i=1}^{l} \frac{T P_{i}}{T P_{i}+F P_{i}}}{l} \ldots \ldots \ldots
$$

The following equation Eq. (5) shows the Recall computation which computed the average per-class effectiveness of the model to identify the judgment of human which yielded about $82.09 \%$.

$$
\text { Recall }=\frac{\sum_{i=1}^{l} \frac{T P_{i}}{T P_{i}+F P_{i}}}{l} \ldots \ldots \ldots
$$

The evaluation of service departments depends upon the sentiment of customer comments in Facebook social networks. So the following rules illustrate the assessment of service departments that depends on sentiment analysis results:

If (Positive Sentiment $\geq 80 \%$ ) then Assessment is Very Good

If $(80 \% \geq$ Positive Sentiment $\geq 70 \%)$ then Assessment is Good

If $(70 \% \geq$ Positive Sentiment $\geq 60 \%)$ and $(20 \% \geq$ Negative Sentiment $\geq 10 \%)$ then Assessment is Medium

If $(60 \% \geq$ Positive Sentiment $\geq 50 \%)$ and $(30 \% \geq$ Negative Sentiment $\geq 10 \%)$ then Assessment is Acceptance

Otherwise the Assessment is bad 
Through the application of rules above on 13 Iraqi service departments, 11\% from these departments have very good assessment, $18 \%$ from these departments have good assessment, $21 \%$ from these departments have medium assessment, 24\% from these departments have acceptance assessment and $26 \%$ from these departments have bad assessment. Figure (3) illustrates those results.

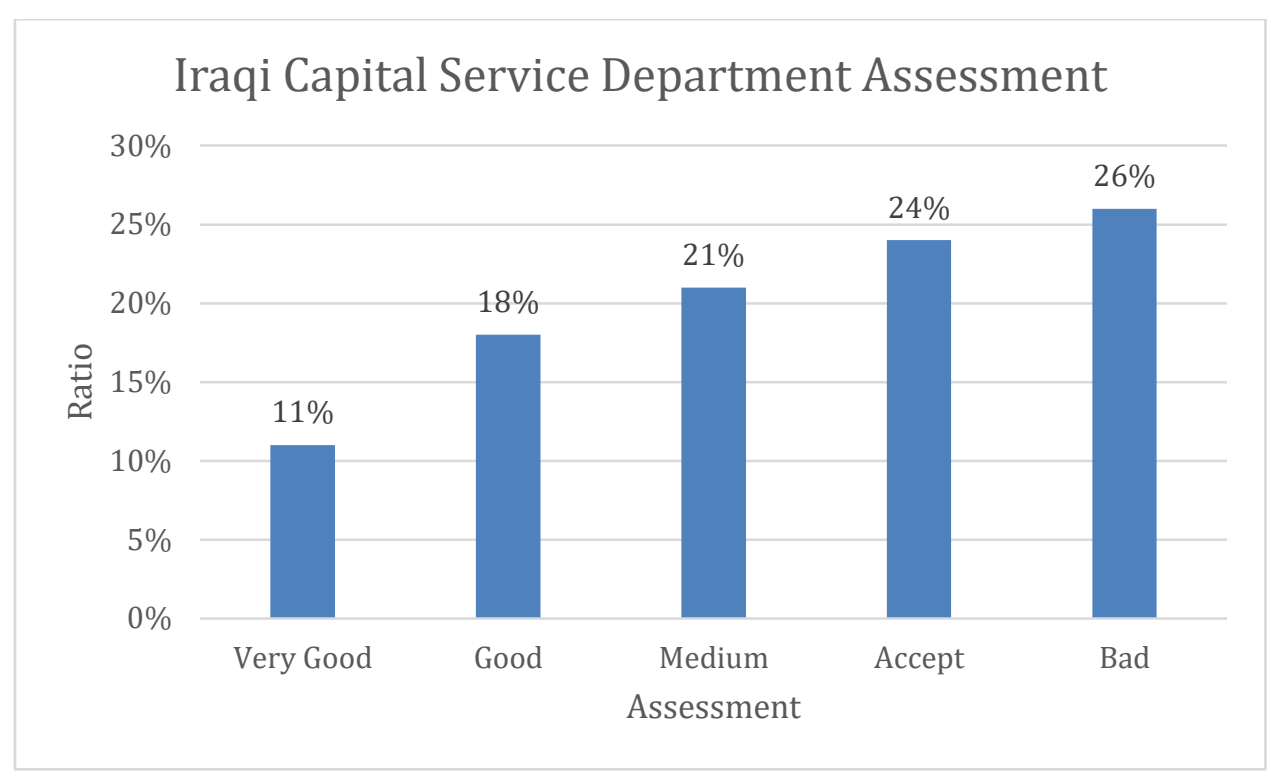

Figure (3) Iraqi Capital Service Department Assessment

\section{Conclusion}

An assessment method that has been based upon the sentiment analyses of the comments of users in Facebook of Iraqi service departments were suggested and implemented. The results of the proposed method represent the real opinions of the users that benefitted from those departments. Standard Arabic language and Iraqi dialect sentiment analysis have a considerable impact on our proposed method. Rough set theory gave the optimal classification accuracy in comparison to NB and KNN, due to the fact that this theory is very beneficial for the categorized data. Rather than the use of the costly researches based on the surveys, the sentiment analysis of user opinions for governmental services resulted in making it easier for the companies to identify their economic value and user opinions about their services that in turn provides insights about the new policies of decision making. The results of the evaluation showed the poor services provided by service departments in the capital, Baghdad. 


\section{REFERENCES}

[1] Al-Bakri, N.F. and Hashim, S.H., 2018. Reducing Data Sparsity in Recommender Systems. Al-Nahrain Journal of Science, 21(2), pp.138-147. DOI: 10.22401/JNUS.21.2.20.

[2] Akaichi, J., Dhouioui, Z. and Pérez, M.J.L.H., 2013, October. Text mining facebook status updates for sentiment classification. In 2013 17th International conference on system theory, control and computing (ICSTCC) pp. 640-645. IEEE.

[3] Alaei, A.R., Becken, S. and Stantic, B., 2019. Sentiment analysis in tourism: capitalizing on big data. Journal of Travel Research, 58(2), pp.175-191.

[4] Junianto, E. and Rachman, R., 2019, November. Implementation of Text Mining Model to Emotions Detection on Social Media Comments Using Particle Swarm Optimization and Naive Bayes Classifier. In 2019 7th International Conference on Cyber and IT Service Management (CITSM) (Vol. 7, pp. 1-6). IEEE.

[5] Loay E. George, Nadia F. Al-Bakri, Nidaa F. Hassan 2015 .An Implementation Of Web Text Mining Method Based On Fuzzy Logic. Journal of Science. Volume 5. Issue 2. 96-101.

[6] Hamouda, A.E.D.A. and El-taher, F.E.Z., 2013. Sentiment Analyzer for Arabic Comments System. Int. J. Adv. Comput. Sci. Appl, 4(3).

[7] Udaruth, S., Moheeputh, S., Permessur, N. and Chamroo, A., 2018. Sentiment Analysis from Facebook Comments using Automatic Coding in NVivo 11. ADCAIJ: Advances in Distributed Computing and Artificial Intelligence Journal, 7(1), pp 41-48.

[8] Ye, Q., Zhang, Z. and Law, R., 2009. Sentiment classification of online reviews to travel destinations by supervised machine learning approaches. Expert systems with applications, 36(3), pp.6527-6535.

[9] Itani, M., Roast, C. and Al-Khayatt, S., 2017, April. Corpora for sentiment analysis of Arabic text in social media. In 2017 8th international conference on information and communication systems (ICICS) (pp. 64-69). IEEE.

[10] Mohammad, A.H., Alwada'n, T. and Al-Momani, O., 2016. Arabic text categorization using support vector machine, Naïve Bayes and neural network. GSTF Journal on Computing (JoC), 5(1), p.108.

[11] Liu, B. (2010). Sentiment analysis and subjectivity. Handbook of natural language processing, 2, 627-666.

[12] Younis, E. M., 2015. Sentiment analysis and text mining for social media microblogs using open source tools: an empirical study. International Journal of Computer Applications, 112(5).

[13] González-Rodríguez, M.R., Martínez-Torres, M.R. and Toral, S.L., 2014, December. Monitoring travel-related information on social media through sentiment analysis. In 2014 IEEE/ACM 7th International Conference on Utility and Cloud Computing (pp. 636-641). IEEE.

[14] Saif Ali Alsaidi, Ahmed T. Sadeq and Hasanen S. Abdullah,2020 "English Poems Categorization using Text Mining and Rough Set Theory", Bulletin of Electrical Engineering and Informatics, 9( 4).

[15] Hasanin Alwan Malik and Ahmed T. Sadiq, 2019 "Arabic Text Mining and Rough Set Theory For Decision Support System", JACSTR,9(4).

[16] Dhafar Hamed Abd, Ahmed T. Sadiq and Ayad R. Abbas, 2019 "A New framework for Automatic Extraction Polarity and Target of Articles", Applied Computing to Support Industry: Innovation and Technology. ACRIT Communications in Computer and Information Science. Vol. 1174. Springer.

[17] Saif Ali Alsaidi, Ahmed T. Sadiq and Hasanen S. Abdullah,2020 "Wisdom Extraction from English Poems Using Tow-Level Feature Selection with Rough Set Theory", International Journal of Advanced Science and Technology, 29(4).

[18] Dhafar Hamed Abd, Ahmed T. Sadiq and Ayad R. Abbas, 2019 "Political Articles Categorization Based on Different Naive Bayes Models", Applied Computing to Support Industry: Innovation and Technology. ACRIT Communications in Computer and Information Science. Vol. 1174. Springer.

[19] Al-Bakri, N., 2015.Email Filtering Using Bayesian Method. International Journal of Scientific \& Engineering Research, 6(1), ISSN 2229-5518.

[20] Ali A. Tuama and Ahmed T. Sadiq, "Lexicon-Based Sentiment Analysis for Iraqi Vernacular/Arabic Language Using KNN, Naive Bayes and Rough Set Theory", Journal of Advanced Research in Dynamical and Control Systems, 11(11), 2020. 